DOI: $10.24850 / j-t y c a-2022-01-02$

Artículos

\title{
Simulación de las garantías de abastecimiento para las demandas de agua en Mexicali, B.C.
}

\section{Simulation of the supply guarantee for the water demands in Mexicali, B.C.}

Raymundo Rangel-Parra1, ORCID: https://orcid.org/0000-0001-89848494

Xan Neira2, ORCID: https://orcid.org/ 0000-0002-1141-6023

Jorge Dafonte ${ }^{3}$, ORCID: https://orcid.org/0000-0003-4305-1521

${ }^{1}$ Departamento de Ingeniería Agroforestal, Escuela Politécnica Superior, Universidad de Santiago de Compostela, Lugo, España, rangel.ray@hotmail.com

2Departamento de Ingeniería Agroforestal, Escuela Politécnica Superior, Universidad de Santiago de Compostela, Lugo, España, xan.neira@usc.es ${ }^{3}$ Departamento de Ingeniería Agroforestal, Escuela Politécnica Superior, Universidad de Santiago de Compostela, Lugo, España, jorge.dafonte@usc.es 
Tecnología y

Ciencias $\stackrel{\Xi}{\unlhd}$ Aua
2022, Instituto Mexicano de Tecnología del Agua

Open Access bajo la licencia CC BY-NC-SA 4.0

(https://creativecommons.org/licenses/by-nc-sa/4.0/)

Autor para correspondencia: Raymundo Rangel-Parra, rangel.ray@hotmail.com

\section{Resumen}

En este trabajo de investigación se realiza un ejercicio de simulación para las garantías en las demandas de agua en Mexicali, considerando un escenario de control con periodo del 2005 al 2018 y un escenario futuro con proyección del 2020 al 2040. En dichas proyecciones se incluyen las reducciones en las entregas de agua a México estipuladas en el Acta 323 de la CILA. Se utilizó el software de gestión de recursos hídricos AQUATOOL, con el que se evaluaron las garantías en el abastecimiento de agua al escenario de control y al futuro, incluyendo también una nueva demanda industrial con una cantidad de hasta $20 \mathrm{Hm}^{3}$ anuales. Se evaluó esta nueva demanda tanto en el escenario futuro como desde el periodo de control para simular qué podría haber pasado si hubiera existido previamente. El resultado muestra que hay ocasiones en que en el escenario de control se presentan algunos fallos en las garantías de abastecimiento. La actual situación de estrés y presión sobre los recursos hídricos, sumados a los efectos del cambio climático en la cuenca del río Colorado y en especial en la ciudad de Mexicali, hacen poco recomendable agregar nuevas demandas al sistema, ya que esto intensifica la competencia por los recursos hídricos.

Palabras clave: gestión de recursos hídricos, río Colorado, AQUATOOL, garantías de abastecimiento. 
Tecnología y

Ciencias $\approx$ Agua
2022, Instituto Mexicano de Tecnología del Agua

Open Access bajo la licencia CC BY-NC-SA 4.0

(https://creativecommons.org/licenses/by-nc-sa/4.0/)

\section{Abstract}

In this research work, a simulation exercise is carried out for the guarantees in the water demands in Mexicali, considering a control scenario with period from 2005 to 2018 and a future scenario with projection from 2020 to 2040. In said projections the reductions in water deliveries to Mexico stipulated in Minute 323 of the IBWC are included. The AQUATOOL water resources management software has been used, with which the guarantees in the water supply to the control scenario and in the future have been evaluated, also including a new industrial demand with an amount of up to $20 \mathrm{Hm}^{3}$ per year. This new demand was evaluated both in the future scenario and in the control period, to simulate what could have happened if it had existed previously. The result shows that there are occasions in the control scenario in which there are some failures in the supply guarantees. The current situation of stress and pressure on water resources, added to the effects of climate change, in the Colorado River basin and especially in the city of Mexicali, make it unwise to add new demands to the system, since this intensifies competition by water resources.

Keywords: Water resources management, Colorado river, AQUATOOL, supply guarantees.

Recibido: 07/10/2020

Aceptado: $02 / 12 / 2020$ 
Tecnología y

Ciencias $₫$ Agua
2022, Instituto Mexicano de Tecnología del Agua

Open Access bajo la licencia CCBY-NC-SA 4.0

(https://creativecommons.org/licenses/by-nc-sa/4.0/)

\section{Introducción}

El río Colorado llega a México entre los límites fronterizos de California y Arizona de EUA, por donde cruza el territorio mexicano entre las ciudades de Mexicali y San Luis Río Colorado. La imagen del río Colorado en el noroeste de México es la de un cauce seco desde hace más de 50 años. Sin embargo, el volumen de agua acarreado anualmente en el río Colorado en EUA varía alrededor de los $17000 \mathrm{Hm}^{3}$, pero toda el agua es controlada por un sistema de presas en el lado de EUA. Los recursos hídricos entre ambos países se gestionan a través de un Tratado de Aguas Internacionales (TAI), en donde se establece a que México le corresponden $1850 \mathrm{Hm}^{3}$ al año.

A raíz del TAI se crea la Comisión Internacional de Límites y Aguas (CILA) como un organismo binacional, multidisciplinario en el área técnico-diplomático, que funciona como base para la aplicación de los acuerdos que se realicen en las reuniones de la comisión y que quedan plasmados en las Actas. De 1889 a 2017 se han firmado 323 Actas. De esta manera, se desarrolla la gestión internacional del agua en la cuenca del río Colorado. Como parte del proceso de manejo adaptativo del río, ambos países acuerdan Actas que se anexan al TAI. Como lo comentan Hinojosa-Herta y Carrillo-Guerrero (2010), estas Actas contienen generalmente especificaciones o temas no incluidos en el cuerpo del 
Tecnología y

Ciencias $\approx$ Agua
2022, Instituto Mexicano de Tecnología del Agua

Open Access bajo la licencia CC BY-NC-SA 4.0

(https://creativecommons.org/licenses/by-nc-sa/4.0/)

tratado per se, como la calidad del agua, implementación de proyectos de mantenimiento de la infraestructura hidráulica y más recientemente aspectos ambientales. En 2017, con la llamada Acta 323, se han establecido una serie de medidas y proyectos mediante un plan de contingencia e inversión para hacer frente a la escasez del recurso.

El río Colorado es la fuente de vida para el suroeste de Estados Unidos y el noroeste de México, pero presenta unas características particulares en la disponibilidad natural de los recursos hídricos. La cuenca transfronteriza del Colorado abarca un área de más de 630000 km² en donde más del $85 \%$ del volumen de agua disponible es captada en las montañas del estado de Colorado, en un área que cubre tan sólo el $15 \%$ de la cuenca en total (Adler, 2007). El volumen de agua acarreado cada año en el río Colorado varía alrededor de los $17000 \mathrm{Hm}^{3}$; sin embargo, el $70 \%$ del volumen anual fluye en la temporada de deshielo (mayo a julio) (Cohen \& Henges-Jeck, 2001). El total de derechos de agua asignados en la cuenca del río Colorado (en ambos países) es de 21586 $\mathrm{Hm}^{3}$ /año (Luecke et al., 1999), por lo que se tiene una sobreasignación de recursos hídricos de aproximadamente $27 \%$. En estas cantidades no se incluye o considera el caudal ecológico.

La importancia de la gestión en la cuenca hidrográfica del río Colorado es crítica al encontrarse en una región donde las precipitaciones son mínimas en la mayor parte de su territorio, altas temperaturas medias, y un crecimiento poblacional y económico continuo. Además, diversas investigaciones (Christensen, Wood, Voisin, Lerrenmaier, \& Palmer, 2004; Ficklin, Stewart, \& Maurer, 2013; USBR, 2016; Udall \& Overpeck, 2017; Gautam \& Mascaro, 2018) coinciden en que las 
Tecnología y

Ciencias $\stackrel{\Xi}{\Im}$ Aua
2022, Instituto Mexicano de Tecnología del Agua

Open Access bajo la licencia CC BY-NC-SA 4.0

(https://creativecommons.org/licenses/by-nc-sa/4.0/)

proyecciones de cambio climático causarán aumentos en la temperatura media anual, así como disminuciones en la precipitación y escorrentía.

Desde el año 2000, según los datos del United States Bureau of Reclamation (USBR, 2019a), los niveles de la presa Hoover (presa que se usa como nivel de referencia para clasificar la situación de los recursos hídricos en la cuenca) ubicada en el lago Mead, se encuentran por debajo de 327 msnm (2015-2016), considerándose como una condición de escasez. Esto dio origen a que en 2017 la CILA firmara el Acta 323, donde se establecen una serie de medidas y proyectos mediante un plan de contingencia e inversión para hacer frente a la escasez del recurso (CILA, 2017a).

La disponibilidad real del agua del río Colorado en México se realiza a través de la coordinación de la CILA de ambos países y en una agenda planificada con base en un Plan Anual de Operación (AOP, por sus siglas en inglés). La distribución real del agua año con año de la cuenca del Colorado para ambos países se fundamenta en la proyección oficial futura de disponibilidad que realiza el USBR. Se trata de un informe con un estudio de 24 meses con proyecciones para el $1^{\circ}$ de enero de cada año. Las operaciones anuales resultantes para el lago Mead se informan en el AOP para los almacenamientos del río Colorado para el año siguiente (CILA, 2017b).

Actualmente disponemos de herramientas tecnológicas que permiten automatizar la simulación y la optimización, dos enfoques esenciales para el modelado de cuencas hidrográficas. Como lo mencionan McKinney, Cai, Rosegrant, Ringler y Scott (1999), en la 
Tecnología y

Ciencias $\approx$ Agua
2022, Instituto Mexicano de Tecnología del Agua

Open Access bajo la licencia CC BY-NC-SA 4.0

(https://creativecommons.org/licenses/by-nc-sa/4.0/)

simulación el comportamiento de los recursos hídricos se simula en función de un conjunto de reglas que rigen las asignaciones de agua y la operación de la infraestructura, mientras que en la optimización las asignaciones se optimizan en función de una función objetiva y restricciones asociadas.

El objetivo que aborda este trabajo es el de realizar un ejercicio de simulaciones para analizarlas garantías de abastecimiento de agua en las demandas en Mexicali, para un escenario de control y otro a futuro. Con esto se busca brindar una nueva perspectiva sobre cómo las herramientas tecnológicas disponibles son una ayuda esencial para la gestión de los recursos hídricos. Debido a que la disponibilidad real de agua del río Colorado a México se determinan cada año por el AOP del USBR, este trabajo únicamente funciona como un ejercicio para probar un software de gestión y planificación de recursos hídricos, analizar los resultados y su grado de realidad, así como para identificar los retos en la obtención de datos para el área de estudio de Mexicali y Baja California en específico.

\section{Área de estudio}

Mexicali es una ciudad con casi un millón de habitantes que se encuentra en el estado de Baja California (B.C.) al noroeste de México, en la frontera 
con EUA. El clima es cálido seco, muy árido, con temperatura media anual de $22.3^{\circ} \mathrm{C}$ y una muy baja precipitación anual estimada en $76.9 \mathrm{~mm}$, lo que provoca una casi nula aportación natural de agua. Los recursos hídricos superficiales que Ilegan a Mexicali son sólo por el último tramo del recorrido del río Colorado, que varían entre el 80 y $90 \%$ de los 1850 $\mathrm{Hm}^{3}$ anuales del TAI.

El río Colorado llega desde EUA a México entre las ciudades de Mexicali y San Luis Río Colorado, como se muestra en la Figura 1. Otros elementos de importancia para la gestión de los recursos hídricos en el sistema de Mexicali son el Acueducto Río Colorado (ARCT) y la extensión del acuífero del Colorado. El ARCT entró en operación en el año de 1982, cumpliendo con la finalidad de abastecer de agua la demanda urbana de las otras ciudades del estado de Baja California, Tecate, Tijuana, Playas de Rosarito y recientemente también Ensenada. 
Teçnología y

Ciencias $\approx$ Agua
2022, Instituto Mexicano de Tecnología del Agua

Open Access bajo la licencia CC BY-NC-SA 4.0

(https://creativecommons.org/licenses/by-nc-sa/4.0/)

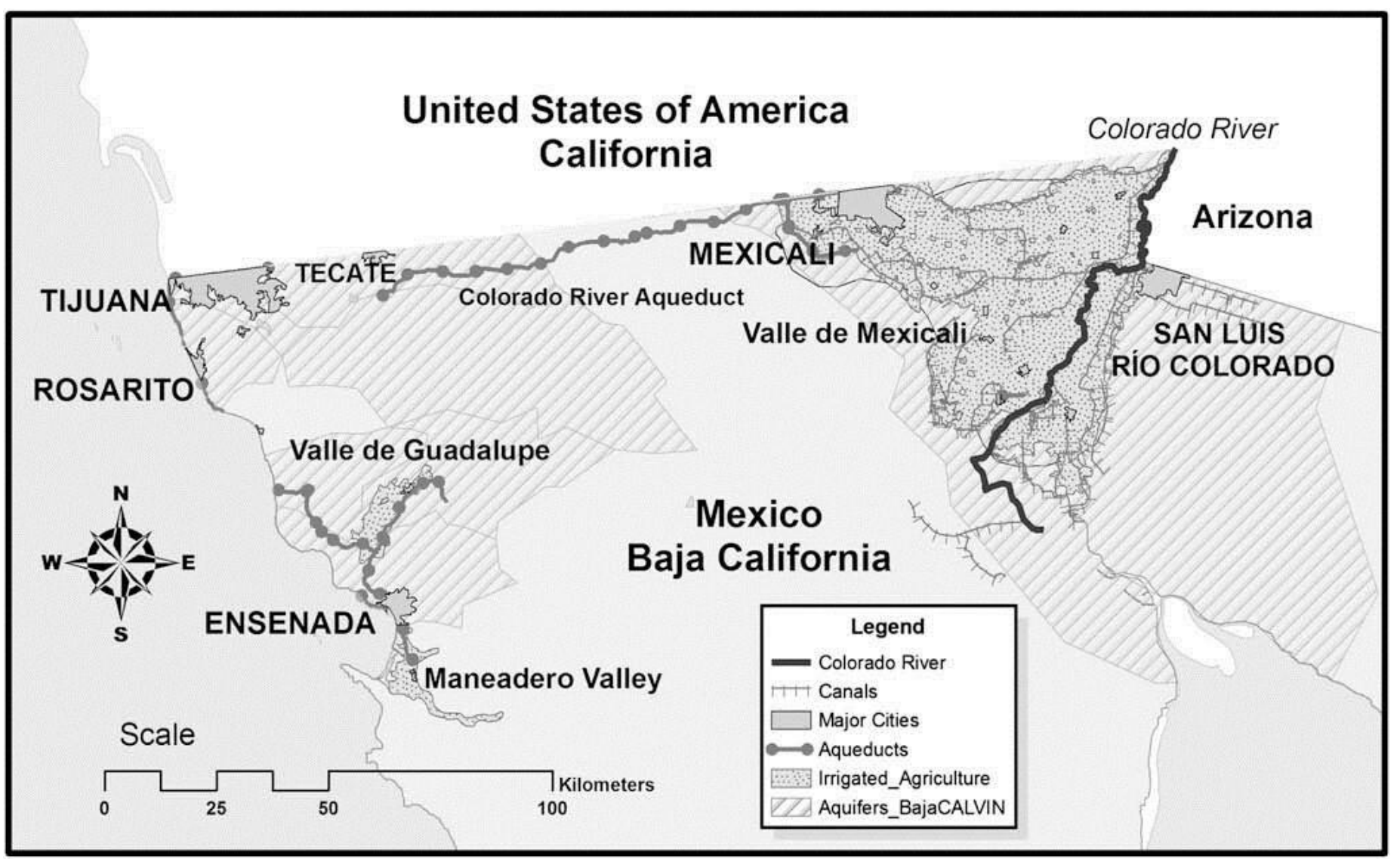

Figura 1. Tramo del río Colorado en la parte mexicana. Fuente:

Medellín-Azuara, Mendoza-Espinosa, Lund, Harou y Howitt (2009).

Del volumen de agua del río Colorado acordado en el TAI se reciben $1677.5 \mathrm{Hm}^{3}$ anuales por el punto denominado Lindero Norte (LN) en la presa derivadora Morelos y $172.7 \mathrm{Hm}^{3}$ por el punto denominado Lindero Sur (LS) en el Canal Sánchez Mejorada.

Respecto a los recursos subterráneos en la parte mexicana, al año se extrae agua con base en las concesiones de pozos federales (500 $\mathrm{Hm}^{3}$ ) 
Tecnología y

Ciencias $\stackrel{\Xi}{\Im}$ Aua
2022, Instituto Mexicano de Tecnología del Agua

Open Access bajo la licencia CC BY-NC-SA 4.0

(https://creativecommons.org/licenses/by-nc-sa/4.0/)

y particulares $\left(200 \mathrm{Hm}^{3}\right.$ ) en los acuíferos 0210-Valle de Mexicali y 2601San Luis Río Colorado, que forman parte del acuífero del Colorado. Asimismo, se tienen asignados volúmenes de agua subterránea para otras ciudades de B.C. (Tijuana, Tecate, Rosarito y Ensenada), que son enviadas por el Acueducto Río Colorado-Tijuana (ARCT) con una extracción de alrededor de $148.96 \mathrm{Hm}^{3}$ anuales. Por lo tanto, también existe una sobre asignación de recursos subterráneos, al ser de $520 \mathrm{Hm}^{3}$ la recarga anual del acuífero. Un aspecto importante es que actualmente no existe un tratado de aguas subterráneas entre México y EUA, además de que existen diferencias entre ambos países respecto a la legislación y jurisdicción de las aguas subterráneas.

Las cantidades de las demandas y origen de los recursos hídricos en el sistema de Mexicali se muestra en la Tabla 1, información que fue recopilada de acuerdo con los escasos datos oficiales encontrados. La demanda mayor se trata de la agrícola en el DR-014, requiriendo alrededor del $90 \%$ de los recursos, seguida del ARCT con más del 5 \% y después la demanda urbana de Mexicali (UDU_Mexicali) con aproximadamente el $2.5 \%$. La demanda anual industrial y comercial (UDI_Mexicali) al 2017 se estimaba en $16.36 \mathrm{Hm}^{3}$ de recurso bruto. También existen tres denominadas demandas rurales (UDR), que corresponden a núcleos de población ubicados en la zona agrícola DR014. Es notable la gran cantidad de agua destinada a la actividad agrícola debido a la gran extensión territorial del DR-014. 
2022, Instituto Mexicano de Tecnología del Agua

Tecnología y

Ciencias $₫$ Agua
Open Access bajo la licencia CCBY-NC-SA 4.0

(https://creativecommons.org/licenses/by-nc-sa/4.0/)

Tabla 1. Resumen de las demandas anuales de agua en el sistema del río Colorado en Mexicali. Elaborado de CEABC (2017) y CEABC (2016).

\begin{tabular}{|c|c|c|c|}
\hline Demanda de agua (2017) & $\begin{array}{c}\text { Superficial } \\
\left(\mathrm{Hm}^{3}\right)\end{array}$ & $\begin{array}{c}\text { Subterránea } \\
\qquad\left(\mathrm{Hm}^{3}\right)\end{array}$ & $\%$ \\
\hline UDU_Mexicali & 68.41 & - & 2.52 \\
\hline UDI_Mexicali & 16.36 & - & 0.60 \\
\hline Pérdidas UDU y UDI & 16.52 & - & 0.61 \\
\hline $\begin{array}{l}\text { Suma } 3 \text { UDR (Valle de } \\
\text { Mexicali) }\end{array}$ & 10.98 & - & 0.40 \\
\hline Demanda agrícola DR-014 & 1753.19 & 700 & 90.38 \\
\hline $\begin{array}{l}\text { Acueducto ARCT (mesa } \\
\text { arenosa) }\end{array}$ & - & 148.96 & 5.49 \\
\hline Subtotal & 1865.46 & 848.9 & \\
\hline Gran total & 27 & 14.36 & 100 \\
\hline
\end{tabular}

A pesar de la limitación natural y del alto grado de presión en los recursos hídricos en Mexicali, aún no existen recortes en el suministro diario de agua. No obstante, esto no quiere decir que el agua esté garantizada para un futuro cercano. Otra característica importante para resaltar es que no existen reservorios de agua de ningún tipo para almacenar recursos. Las condiciones climáticas y de terreno no permiten tener presas más allá de la existente en el límite fronterizo, la Presa 
Tecnología y

Ciencias $₫$ Agua
2022, Instituto Mexicano de Tecnología del Agua

Open Access bajo la licencia CC BY-NC-SA 4.0

(https://creativecommons.org/licenses/by-nc-sa/4.0/)

Morelos, donde los recursos hídricos almacenados no son gestionados a voluntad por México.

Desde 2016 se presentó en Mexicali un conflicto entre grupos sociales y el gobierno del estado debido a la autorización de una nueva demanda industrial. Esta polémica ha llegado hasta escenarios internacionales, donde se cuestiona la disponibilidad de agua para sustentar la demanda industrial declarada en $20 \mathrm{Hm}^{3}$ anuales.

\section{Clima en Mexicali y cambio climático en la cuenca del río Colorado}

En Mexicali se han registrado grandes contrastes térmicos, con temperaturas tan altas como $54.3^{\circ} \mathrm{C}$ y tan bajas como $-7{ }^{\circ} \mathrm{C}$ (SPABC, 2012). Las proyecciones del SRES reportadas por la Secretaría de Protección al Ambiente de B.C. (SPABC) para Mexicali indican que las temperaturas máximas medias anuales para los escenarios A2 y B2 se incrementarán del año 2010 al 2100 en 5 y $4{ }^{\circ} \mathrm{C}$, respectivamente, en comparación con el periodo 1961-1990.

En marzo de 2016, el USBR publicó un reporte técnico de "RECLAMATION - Managing in the West" titulado "West-Wide Climate Risk Assessments: Hydroclimate Projections". Este informe identifica el cambio climático como un riesgo creciente para la gestión del agua en el oeste y 


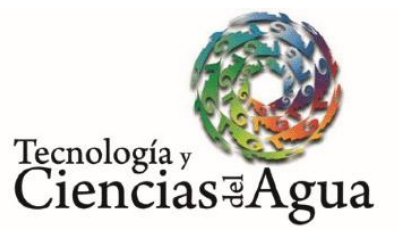

2022, Instituto Mexicano de Tecnología del Agua

Open Access bajo la licencia CC BY-NC-SA 4.0

(https://creativecommons.org/licenses/by-nc-sa/4.0/)

menciona temperaturas más cálidas, cambios en la precipitación, la capa de nieve, y el tiempo y la calidad de la escorrentía en las principales cuencas fluviales como amenazas para la sostenibilidad del agua. Las reducciones en la escorrentía de primavera y principios de verano podrían traducirse en una caída en el suministro de agua para satisfacer las demandas de riego e impactar adversamente las operaciones de energía hidroeléctrica en los embalses de EUA.

Actualmente la gestión del río Colorado enfrenta una situación de sequía que afecta ambos países, los cuales, en 2017, con el Acta 323, han establecido una serie de medidas y proyectos mediante un plan de contingencia e inversión para hacer frente a la escasez del recurso. Se especifica el Plan Binacional de Contingencia, en el cual se marcan dos cosas relevantes: 1 ) las acciones a realizar respecto a niveles altos en el lago Mead con incrementos en la entrega y para niveles bajos con reducciones (para el caso de México sería de 51 a $185 \mathrm{Hm}^{3}$ en el total entregado) en la disponibilidad para ambos países, y 2) los proyectos para conservación y nuevas fuentes de agua tanto en México como en EUA.

\section{Materiales y métodos}




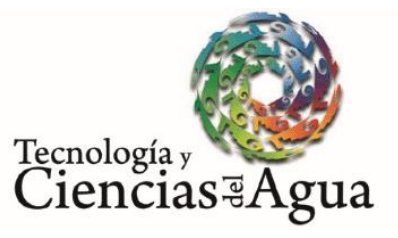

2022, Instituto Mexicano de Tecnología del Agua

Open Access bajo la licencia CCBY-NC-SA 4.0

(https://creativecommons.org/licenses/by-nc-sa/4.0/)

Como herramienta de simulación se utilizó el software AQUATOOL y su módulo SIMGES, desarrollados por la Universidad Politécnica de Valencia, España. Solera-Solera, Paredes-Arquiola y Andreu-Álvarez (2015) lo describen como una interface o entorno de trabajo para el desarrollo y análisis de sistemas de ayuda en la decisión de planificación y gestión de cuencas. El programa es una serie de fichas interactivas que permiten la edición de los datos necesarios para el análisis de alternativas de gestión de los recursos hídricos de cuencas hidrográficas.

En AQUATOOL, la simulación y gestión del sistema superficial se efectúan a un tiempo mediante el uso de un algoritmo de optimización de redes de flujo conservativo. Dicho algoritmo se encarga de determinar el flujo en el sistema, tratando de satisfacer al máximo los objetivos múltiples de minimización de déficits (Andreu, Solera, Capilla, \& Ferrer, 2007). Por tanto, la primera tarea del modelo después de la lectura de datos de entrada es la adaptación del esquema del usuario como una red de flujo conservativo. Para ello es necesario el desdoblamiento de cada elemento del sistema. El resultado es una "red de flujo interna" mucho más compleja que la del usuario, que ya es conservativa, y que es la que manejará el modelo sin trascendencia exterior al usuario. Una vez confeccionada dicha red interna se entra en la dinámica de la simulación, en la que para cada mes del periodo de simulación se resuelve la red de flujo con los valores de aportaciones, demandas y parámetros de gestión correspondientes a ese mes; se itera entre estos dos últimos pasos, y se almacenan valores para su escritura anual y estadísticas. Finalmente, una vez terminado el periodo de simulación, se procede a la confección de estadísticas y cálculos de garantías (Andreu et al., 2007). 
Tecnología y

Ciencias $₫$ Agua
2022, Instituto Mexicano de Tecnología del Agua

Open Access bajo la licencia CCBY-NC-SA 4.0

(https://creativecommons.org/licenses/by-nc-sa/4.0/)

Los criterios que utiliza AQUATOOL para evaluar las garantías han sido los de la instrucción para la planificación hidrológica (IPH) del 2008 (IPH, 2008) (BOE, Orden ARM/2656/2008) del gobierno de España para demandas urbanas y agrícolas (criterio UTAH DWR):

"Para la demanda urbana, se considera fallo cuando en el déficit en un mes es superior al $10 \%$ de la demanda mensual. Para la demanda agrícola, se considera fallo cuando el déficit en un año es superior al $50 \%$ de la demanda anual. (D.A)., se considera fallo cuando en dos años consecutivos la suma del déficit es superior al $75 \%$ de la D.A., y se considera fallo cuando en diez años consecutivos la suma de déficit es superior al $100 \%$ de la D.A.".

\section{Esquema para la simulación del agua en el sistema de Mexicali}

La disponibilidad del recurso hídrico del río Colorado para México está determinada por el TAI de 1944; la operación de las entregas es estimada por el USBR en toda la cuenca en general, y los flujos son monitoreados por la CILA (2018). Por tal motivo, no corresponde realizar la simulación 
Tecnología y

Ciencias $\approx$ Agua
2022, Instituto Mexicano de Tecnología del Agua

Open Access bajo la licencia CC BY-NC-SA 4.0

(https://creativecommons.org/licenses/by-nc-sa/4.0/)

hidrológica en la cuenca del río Colorado aguas arriba del punto de entrega a México para conocer el volumen de agua disponible para Mexicali mensualmente. Este volumen de agua se puede conocer con los datos de las entregas de los últimos años en el LIN y así obtener un promedio de entrega para cada mes, que se puede considerar como la disponibilidad al mes correspondiente. La cantidad de agua retenida en el LIN no es manejada por México sino a través de las negociaciones diplomáticas entre la CILA de ambos países.

Se planteó realizar una serie de simulaciones para analizar la garantía del abastecimiento de las demandas actuales y futuras en Mexicali. El Acta 323 estipula una serie de reducciones a los $1850 \mathrm{Hm}^{3}$ en caso de que los niveles de la presa Hoover (lago Mead) continúen descendiendo. De acuerdo con las proyecciones del USBR y las cantidades establecidas en el Acta 323, en 2017 se debió aplicar la primera reducción de $51 \mathrm{Hm}^{3}$ y en 2019 la segunda reducción adicional de $37 \mathrm{Hm}^{3}$. Utilizando una caída lineal de los niveles de la presa, un factor de variación mensual en las entregas de agua a México y las adicionales reducciones aplicables según el Acta 323, se calcularon las entregas de agua reducidas a México hasta 2040. Para estas proyecciones al 2040 se asumen las condiciones futuras en la cuenca del río Colorado que Ilevaran a una reducción de los recursos hídricos, como lo concuerdan los estudios de proyección climática realizados en la cuenca del Colorado.

En lo que respecta a consideraciones especiales para definiralgunas demandas, el ARCT ha sido como una de ellas, pues el agua que se bombea por el acueducto está en función de una demanda y de esta manera se pueden evaluar la garantía y los fallos. 
Tecnología y

Ciencias $₫$ Agua
2022, Instituto Mexicano de Tecnología del Agua

Open Access bajo la licencia CC BY-NC-SA 4.0

(https://creativecommons.org/licenses/by-nc-sa/4.0/)

Al momento de la realización de este trabajo (2017-2018), no se encontró información detallada sobre la distribución exacta en tiempo y espacio de la demanda agrícola, ni sobre los recursos hídricos subterráneos utilizados principalmente en el DR-014 ubicado en el Valle de Mexicali. Ante esta situación, se optó por hacer una estimación indirecta con la información de las entregas de agua en el LIN y LIS, pues se entiende que la entrega debe estar en función de una demanda. Con una estimación de los promedios de entrega de agua por cada mes se obtiene un factor de variación temporal que, multiplicado por del total del uso anual de la demanda agrícola declarado por Conagua (2018), se calcula el uso de agua para cada mes.

\section{Escenario del sistema de Mexicali con una nueva demanda de agua}

Con el fin de evaluar el escenario futuro, se agregó al sistema de flujos de agua de Mexicali una nueva demanda industrial. El volumen de 20

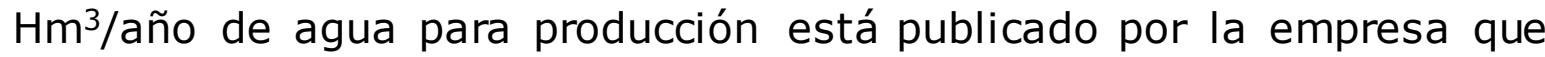
requiere la demanda de agua (en su página web http://www.cbrands.mx/nuestra-historia/unidades-de-negocio). 
Tecnología y

Ciencias ฐึgua
2022, Instituto Mexicano de Tecnología del Agua

Open Access bajo la licencia CC BY-NC-SA 4.0

(https://creativecommons.org/licenses/by-nc-sa/4.0/)

Los escenarios establecidos para las simulaciones consistieron en un periodo de control con datos reales de entregas de agua (2005-2020) y un escenario futuro (2020-2040) con las proyecciones de las entregas de agua reducidas para México. Con estos escenarios se simularon las garantías en el abastecimiento de las demandas actuales de Mexicali y hasta el 2040.

Posteriormente, en otra simulación separada, se agregó la nueva demanda industrial de $20 \mathrm{Hm}^{3}$ tanto en el escenario de control como en el futuro, para evaluar su comportamiento en un periodo de datos reales y con datos proyectados, respectivamente. Esta nueva demanda fue planeada en dos fases: una de $5 \mathrm{Hm}^{3}$ y otra de $20 \mathrm{Hm}^{3}$, ya que así estaba planeada la operación industrial que iniciaría con 5 y aumentaría hasta 20. La fuente de abastecimiento para esta nueva demanda industrial es de los recursos superficiales, pues sólo se puede evaluar el impacto que tendría en estos recursos al ser los únicos de los que se tienen información más o menos detallada debido a que los datos sobre las extracciones de agua subterránea son mínimos o ausentes.

\section{Resultados}


Teçnología y

Ciencias $\stackrel{\Xi}{\unlhd}$ Aua
2022, Instituto Mexicano de Tecnología del Agua

Open Access bajo la licencia CC BY-NC-SA 4.0

(https://creativecommons.org/licenses/by-nc-sa/4.0/)

El esquema en la Figura 2 representa de manera simplificada el flujo de agua en el sistema en la ciudad de Mexicali, pues el esquema en AQUATOOL es demasiado extenso para ser representado en este documento. El esquema se hizo con base en las fuentes de información disponible en informes y publicaciones de la CEABC y la CILA, así como de los estudios hechos en EUA que involucran a la parte del río Colorado en Mexicali. Se empleó la orientación de los esquemas de flujos en las investigaciones de Medellín-Azuara et al. (2009); Howes, Burt y Feist (2012), y Carrillo-Guerrero, Glenn e Hinojosa-Huerta (2013).
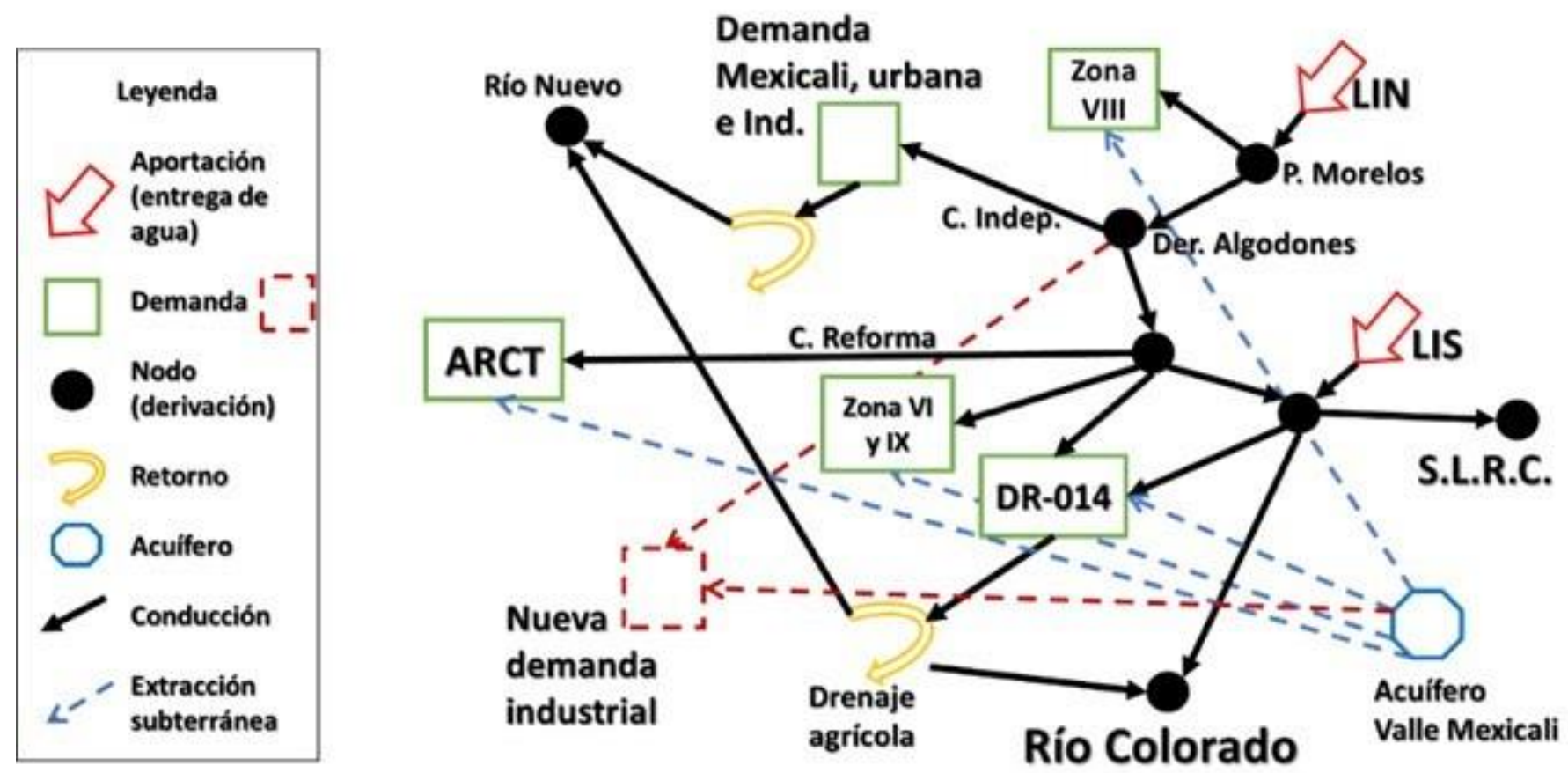

Figura 2. Esquema "simplificado" de AQUATOOL para el sistema de flujos de agua en Mexicali. 
Tecnología y

Ciencias $\stackrel{\unlhd}{ }$ Agua
2022, Instituto Mexicano de Tecnología del Agua

Open Access bajo la licencia CCBY-NC-SA 4.0

(https://creativecommons.org/licenses/by-nc-sa/4.0/)

En la Tabla 2 y en la Figura 3 se presenta la estadística mensual, promedio, mínima, máxima, desviación estándar y porcentaje de distribución de las entregas de agua del río Colorado a México en el periodo 2005-2018. Se calculó un Factor de Variación en el Tiempo (FVT) (Ecuación (1)), para poder calcular otras demandas de las que no se tienen más que solamente cantidades anuales, como es el caso de la demanda agrícola. Se puede observar que en marzo y abril es cuando México recibe la mayor cantidad de agua y en octubre la menor cantidad.

$F V T=\frac{\overline{Q_{l}}}{\sum_{i=1}^{n} \overline{Q_{l}}}$

En donde " $i$ " es el mes y " $n$ " es el número de meses total de meses " $i$ ". En este caso, "Q" es el promedio de cada mes en el periodo 20052015.

Tabla 2. Estadística mensual de entregas de agua a México 2005-2018 y FVT.

\begin{tabular}{|c|c|c|c|c|c|}
\hline \multicolumn{6}{|c|}{ Promedio mensual de entregas de agua a México } \\
\hline & $(\mathbf{Q}) \mathbf{H m}^{\mathbf{3}}$ & $\mathbf{H m}^{\mathbf{3}}$ & $\mathbf{H m}^{\mathbf{3}}$ & $\mathbf{H m}^{\mathbf{3}}$ & \% (FVT) \\
\hline Mes (i) & Promedio & Mín & Máx & $\begin{array}{c}\text { Desv. } \\
\text { est }\end{array}$ & Distribució \\
& & & & $\mathrm{n}$ \\
\hline
\end{tabular}


2022, Instituto Mexicano de Tecnología del Agua

Tecnología y

Ciencias $\stackrel{\unlhd}{\unlhd}$ gua
Open Access bajo la licencia CC BY-NC-SA 4.0

(https://creativecommons.org/licenses/by-nc-sa/4.0/)

\begin{tabular}{|l|c|c|c|c|c|}
\hline Ene & 167.39 & 147.50 & 214.62 & 17.38 & 0.089 \\
\hline Feb & 196.87 & 173.02 & 229.09 & 16.25 & 0.105 \\
\hline Mar & 253.94 & 220.10 & 286.66 & 16.22 & 0.136 \\
\hline Abr & 240.32 & 211.85 & 258.21 & 14.64 & 0.128 \\
\hline May & 133.86 & 104.17 & 152.07 & 11.82 & 0.072 \\
\hline Jun & 138.37 & 105.68 & 166.47 & 15.63 & 0.074 \\
\hline Jul & 147.74 & 119.75 & 163.40 & 12.19 & 0.079 \\
\hline Ago & 125.00 & 114.53 & 145.22 & 7.35 & 0.067 \\
\hline Sep & 114.32 & 109.79 & 127.05 & 4.17 & 0.061 \\
\hline Oct & 89.88 & 72.11 & 130.34 & 16.40 & 0.048 \\
\hline Nov & 118.96 & 98.25 & 142.35 & 11.81 & 0.064 \\
\hline Dic & 145.28 & 109.52 & 173.11 & 19.36 & 0.078 \\
\hline Total & 1871.93 & & & & 1 \\
\hline
\end{tabular}




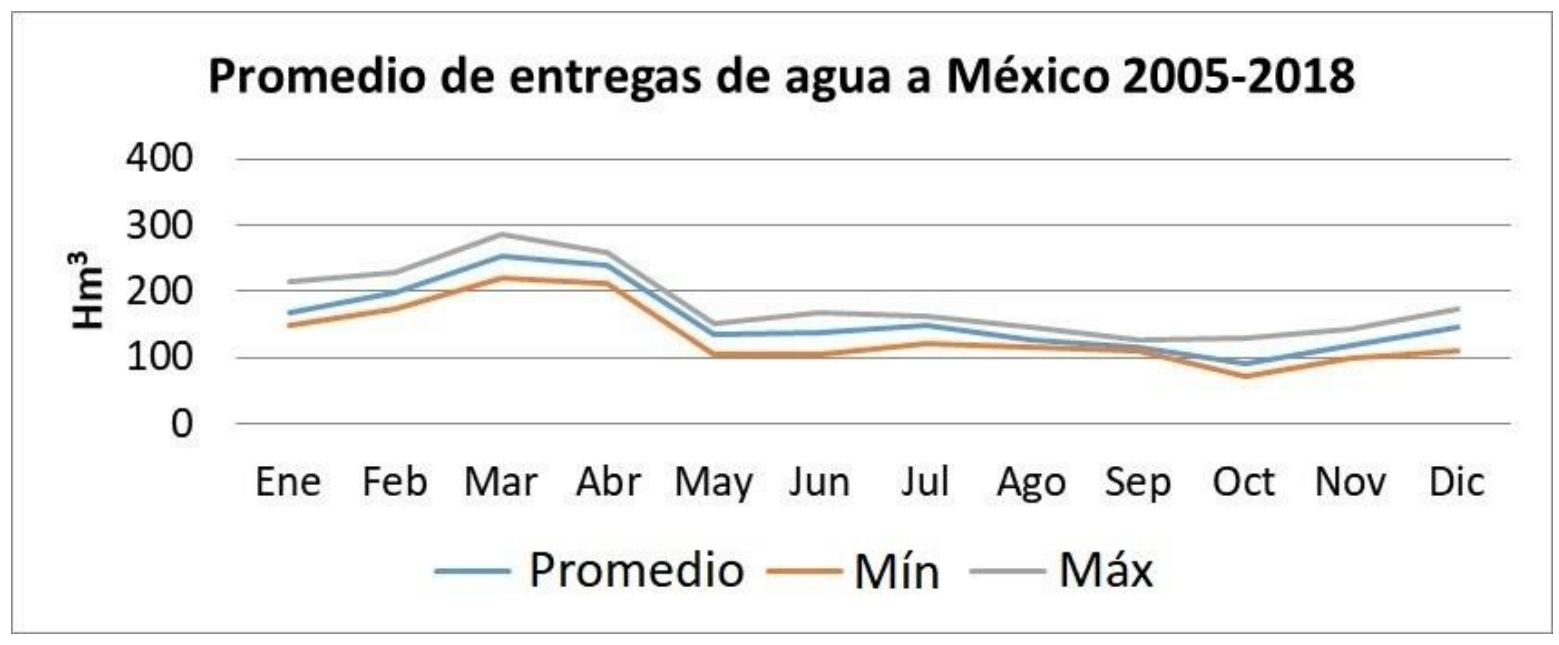

Figura 3. Promedio, mínima, máxima mensual de las entregas de agua del río Colorado a México, 2005-2018.

En ausencia de datos mensuales de la demanda agrícola, se usó un cálculo indirecto para determinarla, utilizando el FVT de la Tabla 2 y multiplicándolo por la cantidad anual reportada para 2015-2016 (1 450 $\mathrm{Hm}^{3}$ superficial y $700 \mathrm{Hm}^{3}$ subterránea) en esta demanda agrícola según los datos de la Conagua (2018). Como se muestra en la Tabla 3, de esta manera se puede obtener una distribución mensual de la demanda agrícola de la que no hay disponibles datos mensuales. 
2022, Instituto Mexicano de Tecnología del Agua

Tecnología y Open Access bajo la licencia CC BY-NC-SA 4.0

(https://creativecommons.org/licenses/by-nc-sa/4.0/)

Tabla 3. Distribución mensual de la demanda agrícola en el DR-014.

\begin{tabular}{|l|c|c|c|c|}
\hline \multicolumn{1}{|c|}{ Mes } & $\begin{array}{c}\text { FVT en } \\
\text { entregas } \\
\text { LIN y } \\
\text { LIS }\end{array}$ & $\begin{array}{c}\text { Demanda } \\
\text { superficial } \\
\mathbf{H m}^{3}\end{array}$ & $\begin{array}{c}\text { Demanda } \\
\text { subterránea } \\
\text { Hm }^{3}\end{array}$ & $\begin{array}{c}\text { Demanda } \\
\text { total } \\
\text { DR014 } \\
\text { Hm }^{3}\end{array}$ \\
\hline Enero & 0.089 & 129.67 & 62.594 & 192.262 \\
\hline Febrero & 0.105 & 152.50 & 73.618 & 226.122 \\
\hline Marzo & 0.136 & 196.71 & 94.959 & 291.673 \\
\hline Abril & 0.128 & 186.17 & 89.867 & 276.034 \\
\hline Mayo & 0.072 & 103.70 & 50.058 & 153.756 \\
\hline Junio & 0.074 & 107.19 & 51.741 & 158.927 \\
\hline Julio & 0.079 & 114.45 & 55.247 & 169.694 \\
\hline Agosto & 0.067 & 96.83 & 46.744 & 143.578 \\
\hline Septiembre & 0.061 & 88.56 & 42.749 & 131.306 \\
\hline Octubre & 0.048 & 69.62 & 33.610 & 103.235 \\
\hline Noviembre & 0.064 & 92.15 & 44.485 & 136.640 \\
\hline Diciembre & 0.078 & 112.54 & 54.328 & 166.873 \\
\hline Total & 1.000 & 1450.100 & 700.000 & 2150.100 \\
\hline
\end{tabular}

En la Figura 4 se presenta la gráfica con las distribuciones de la demanda agua de Mexicali (suma de la demanda urbana, industrial y rural), la demanda agrícola y la demanda del acueducto que va para la 
Teçnología y

Ciencias $\stackrel{\unlhd}{\unlhd}$ gua
2022, Instituto Mexicano de Tecnología del Agua

Open Access bajo la licencia CCBY-NC-SA 4.0

(https://creativecommons.org/licenses/by-nc-sa/4.0/)

ciudad de Tijuana y otras ciudades del estado de Baja California. Se puede observar la proporción mucho mayor de la demanda agrícola en comparación de las otras dos.

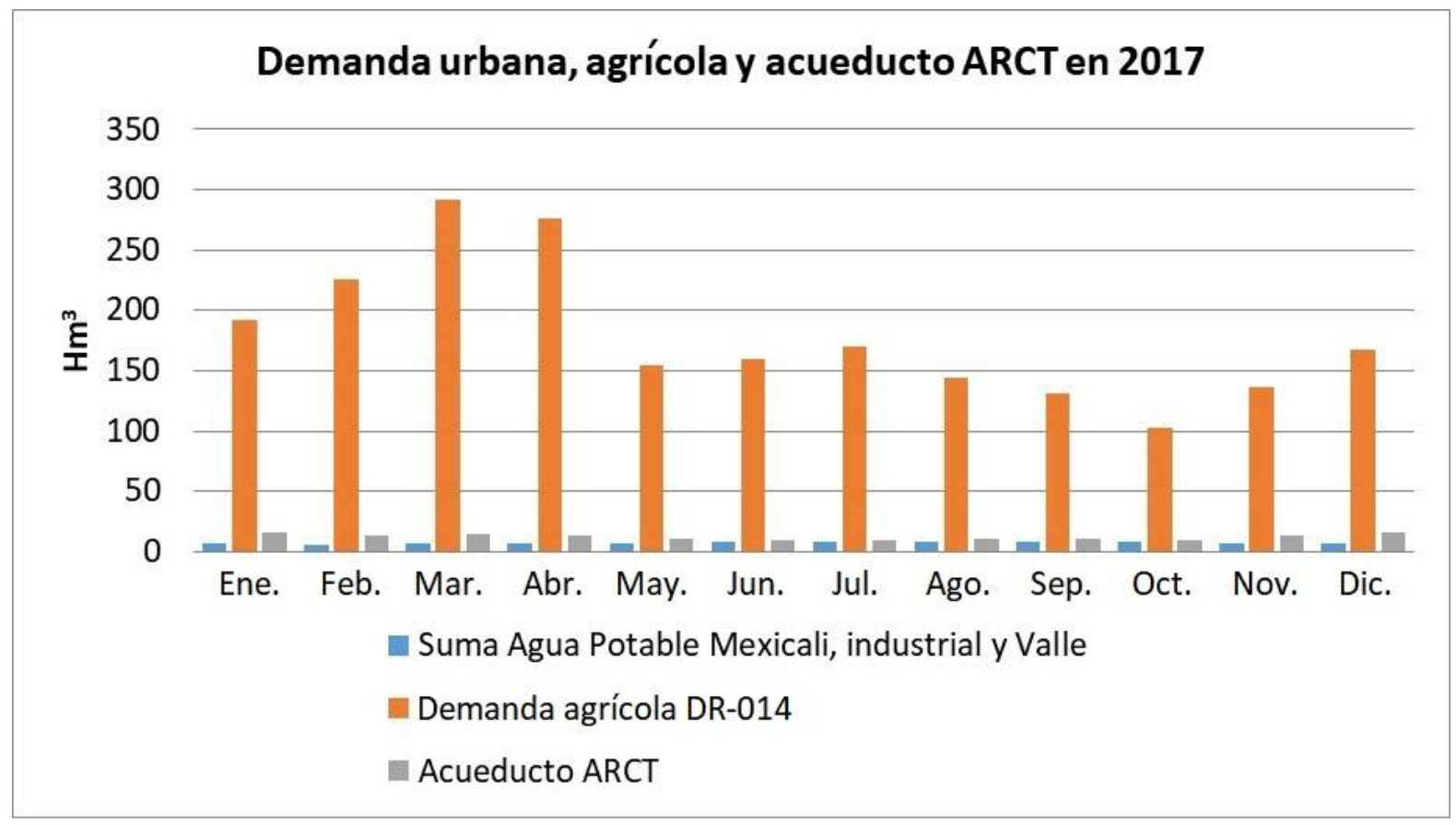

Figura 4. Demanda mensual de agua urbana, agrícola y del Acueducto ARCT para el 2017 en Mexicali.

Con las especificaciones de las reducciones aplicables establecidas en el Acta 323, los niveles proyectados al 2040 de la presa Hoover y las entregas de agua a México observadas del periodo 2005-2018, se han estimado las proyecciones de entregas de agua hasta el 2040. En la Figura 5 se representa la gráfica de las proyecciones, en donde las reducciones iniciarían en octubre de 2017 con $51 \mathrm{Hm}^{3}$ por año, restados al total anual 


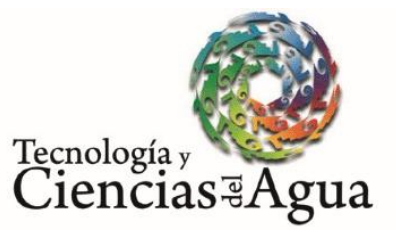

2022, Instituto Mexicano de Tecnología del Agua

Open Access bajo la licencia CC BY-NC-SA 4.0

(https://creativecommons.org/licenses/by-nc-sa/4.0/)

de los derechos de $1850 \mathrm{Hm}^{3}$, hasta que el nivel de la presa de referencia suba o baje a la siguiente categoría. Esta primera reducción significa en realidad una disminución mensual entre 1.49 y $4.20 \mathrm{Hm}^{3}$ según el mes. Posteriormente, las siguientes adicionales reducciones (ya que son acumulativas) se estiman en mayo de 2019 con $37 \mathrm{Hm}^{3}$ y hasta julio de 2026, cuando se alcanzaría la máxima reducción estipulada en el Acta 323 de $185 \mathrm{Hm}^{3}$. Para dar presencia a las predicciones de los efectos del cambio climático en la cuenca del río Colorado estimados por la USBR y los otros estudios mencionados que coinciden en una disminución de la disponibilidad de los recursos hídricos en toda la cuenca, se aplicó una reducción a las entregas de agua siguiendo la tendencia de la tabla establecida en el Acta hasta llegar al año 2040. Las entregas de agua a México tanto del periodo observado como del proyectado son consideradas como las aportaciones que fueron ingresadas al software AQUATOOL. 
Teçnología y

Ciencias $\stackrel{\unlhd}{\unlhd}$ gua
2022, Instituto Mexicano de Tecnología del Agua

Open Access bajo la licencia CC BY-NC-SA 4.0

(https://creativecommons.org/licenses/by-nc-sa/4.0/)

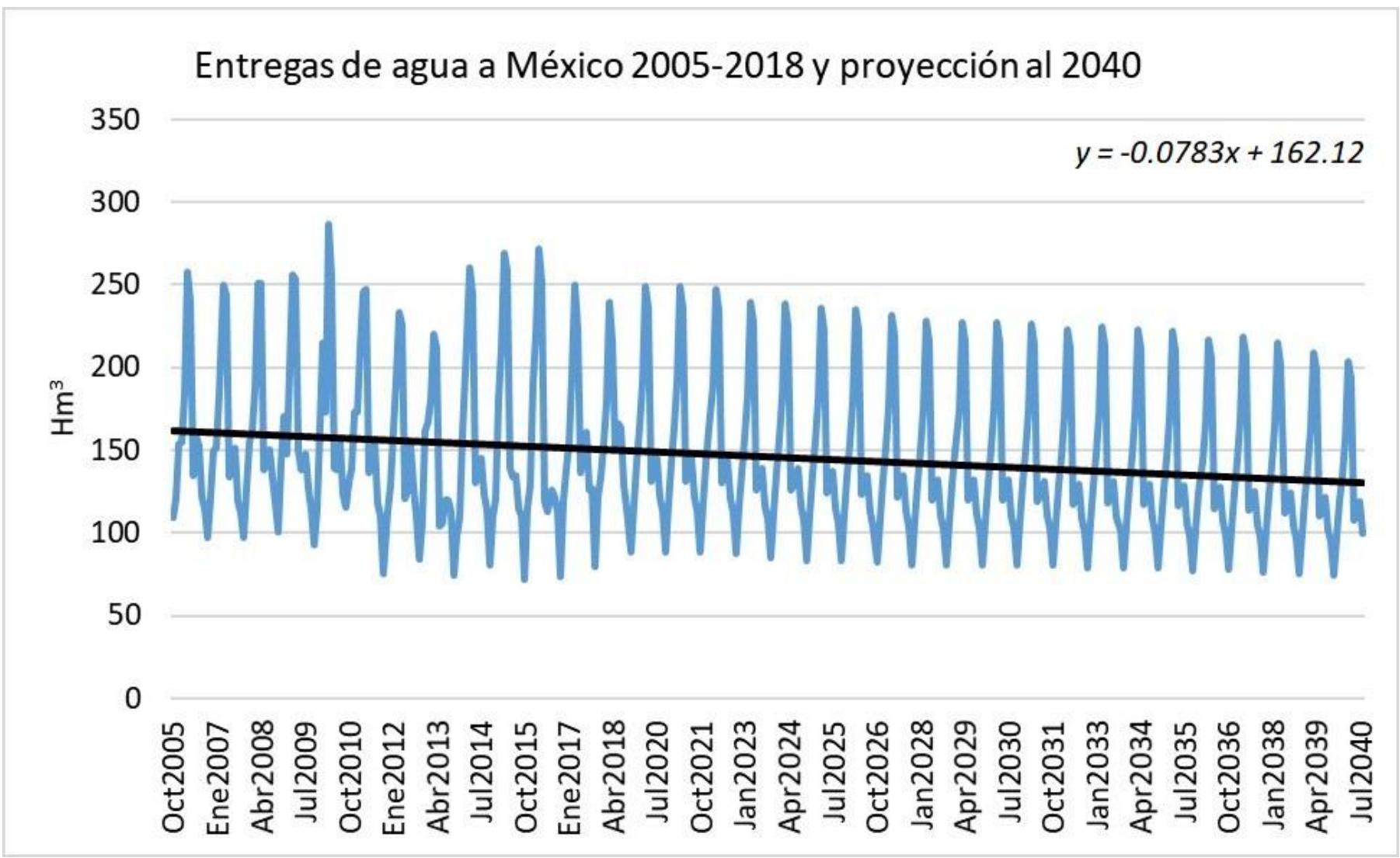

Figura 5. Entrega real mensual de agua México 2005-2018 y proyección con reducciones al 2040.

\section{Simulaciones con las demandas actuales}

Una vez que se estimaron la distribución de la demanda de agua agrícola y las aportaciones proyectadas para el escenario futuro 2020-2040 fue 
Tecnología y

Ciencias $\stackrel{\Xi}{\Im}$ Aua
2022, Instituto Mexicano de Tecnología del Agua

Open Access bajo la licencia CC BY-NC-SA 4.0

(https://creativecommons.org/licenses/by-nc-sa/4.0/)

posible realizar las simulaciones tanto en el escenario de control como en el futuro. El periodo simulado en AQUATOOL corresponde a 35 años hidrológicos de 2005 a 2040. Para el orden de prioridad de abastecimiento en el software AQUATOOL se ha dado la mayor importancia a las demandas urbanas e industriales (incluyendo el ARCT) sobre la demanda agrícola.

Los resultados en las simulaciones con AQUATOOL muestran que sólo la demanda agrícola DR-014 presenta nueve fallos en la garantía de abastecimiento en el escenario 2005-2020. Después, esta misma demanda más la del acueducto ARCT y dos demandas rurales presentan aún más fallos para el periodo futuro 2020-2040 con reducciones de agua. Estos fallos en el abastecimiento agrícola se puede pensar que muy probablemente fueron satisfechos por una mayor extracción de agua subterránea por parte de los usuarios o alguna medida de adaptación de riego. Sin embargo, no existen datos públicos de un control exacto sobre la extracción de agua subterránea. Para el escenario de 2020-2040, con solamente las demandas de agua actuales, se presentan 18 fallos en el DR-014 generados por las disminuciones calculadas respecto a las disminuciones en las entregas a México hasta 2040. El acueducto ARCT presenta 18 fallos en el periodo 2020-2040, aunque a pesar de esto, las garantías parecen estar por encima del 90 \%. Las demandas rurales UDR_Zona_VIII y Zona_IX presentan un total de 12 fallos en el periodo 2020-2040. Incluso con estos fallos estimados, las ga rantías volumétricas en estas demandas de agua se mantienen por encima del 97 \% (Tabla 4). 
2022, Instituto Mexicano de Tecnología del Agua

Tecnología y

Ciencias $\stackrel{\Xi}{\unlhd}$ gua
Open Access bajo la licencia CC BY-NC-SA 4.0

(https://creativecommons.org/licenses/by-nc-sa/4.0/)

Tabla 4. Resultado de fallos en la simulación de las garantías 20052020 y 2020-2040 en el sistema del río Colorado en Mexicali.

\begin{tabular}{|l|c|c|c|c|c|c|}
\hline \multicolumn{1}{|c|}{ Demanda } & $\begin{array}{c}\text { Núm. } \\
\text { fallos } \\
\mathbf{2 0 0 5 -} \\
\mathbf{2 0 2 0}\end{array}$ & $\begin{array}{c}\text { Núm. } \\
\text { fallos } \\
\mathbf{2 0 2 0} \\
\mathbf{2 0 4 0}\end{array}$ & $\begin{array}{c}\text { Garantía } \\
\mathbf{( \% )}\end{array}$ & $\begin{array}{c}\text { Garantía } \\
\text { volumé- } \\
\text { trica (\%) }\end{array}$ & $\begin{array}{c}\text { Máx. } \\
\text { déficit } \\
\text { mensual } \\
\mathbf{( H m}^{\mathbf{3}} \mathbf{)}\end{array}$ & $\begin{array}{c}\text { Máx. } \\
\text { déficit } \\
\text { en dos } \\
\text { meses } \\
\mathbf{( H m ^ { 3 }}\end{array}$ \\
\hline UDA_DR-014 & 9 & 18 & 93.6 & 98.9 & 94.96 & 107.58 \\
\hline ARCT & 0 & 18 & 95.7 & 97.3 & 14.659 & 25.28 \\
\hline UDR_ZONA_V & 0 & 12 & 97.1 & 97.2 & 0.421 & 0.726 \\
\hline $\begin{array}{l}\text { UDR_ZONA_I } \\
\text { X }\end{array}$ & 0 & 12 & 97.1 & 99.3 & 0.287 & 0.391 \\
\hline
\end{tabular}

\section{Simulaciones con la nueva demanda de agua industrial}

Se agregó la supuesta nueva demanda de agua industrial al sistema de flujos de agua en Mexicali para evaluar el comportamiento y determinar si existirían fallos en cumplir con su garantía. En el esquema de AQUATOOL se agregó la nueva demanda en dos fases. La primera fase se abastece de $5 \mathrm{Hm}^{3} /$ año y la segunda con $20 \mathrm{Hm}^{3}$, siendo ambas de fuente 
de agua superficial. Las dos fases se evaluaron desde el periodo de datos observados hasta la proyección futura.

Los resultados de las simulaciones observados en la Figura 6 muestran que si bien los primeros años del escenario futuro 2020-2040 no se presentan fallos en el abastecimiento, sí se presentan hacia el final del periodo, coincidiendo con los fallos de las otras demandas y con los respectivos aumentos en los recortes de entrega de agua. Además, la nueva demanda industrial también presenta fallos en el periodo 20052020 tanto para la fase de $5 \mathrm{Hm}^{3}$ como de $20 \mathrm{Hm}^{3}$. Esto significaría que, en ciertos años, si la nueva demanda hubiera existido en el periodo de datos observados, habría presentado fallos en su abastecimiento y, por consiguiente, una competencia con las otras demandas reales. 
Ciencias $\stackrel{2}{\mathrm{v}}$ gua
2022, Instituto Mexicano de Tecnología del Agua

Open Access bajo la licencia CC BY-NC-SA 4.0

(https://creativecommons.org/licenses/by-nc-sa/4.0/)

\section{A) Fallos en la demanda de $5 \mathrm{Hm}^{3} /$ año}

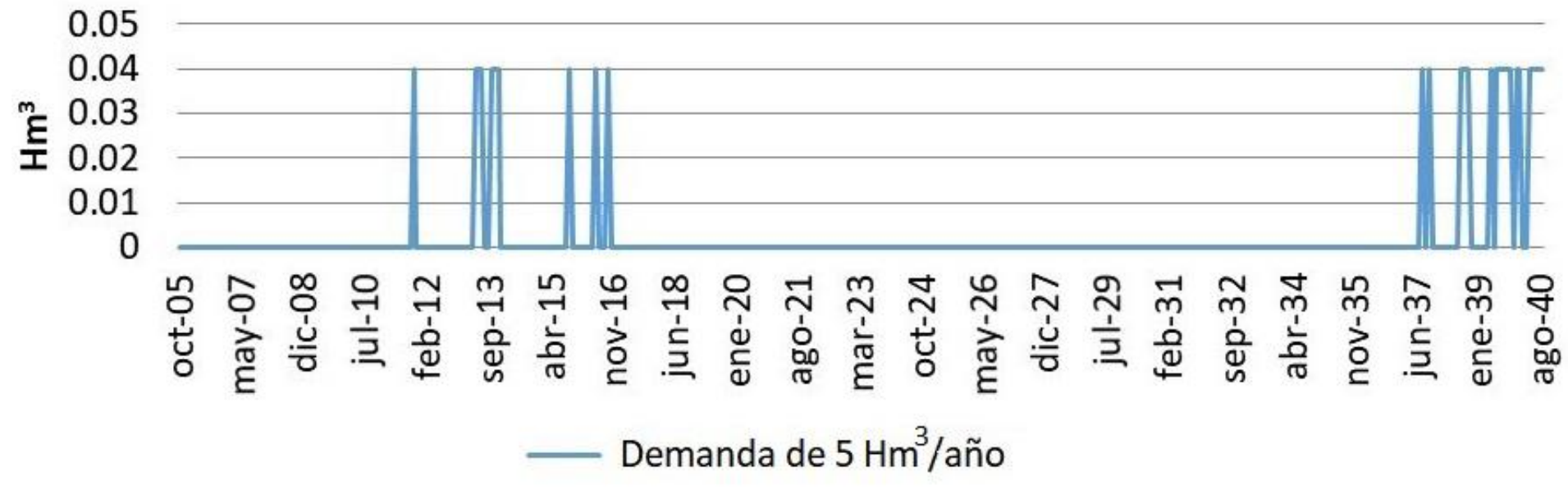

\section{B) Fallos en la demanda de $20 \mathrm{Hm}^{3}$ /año}

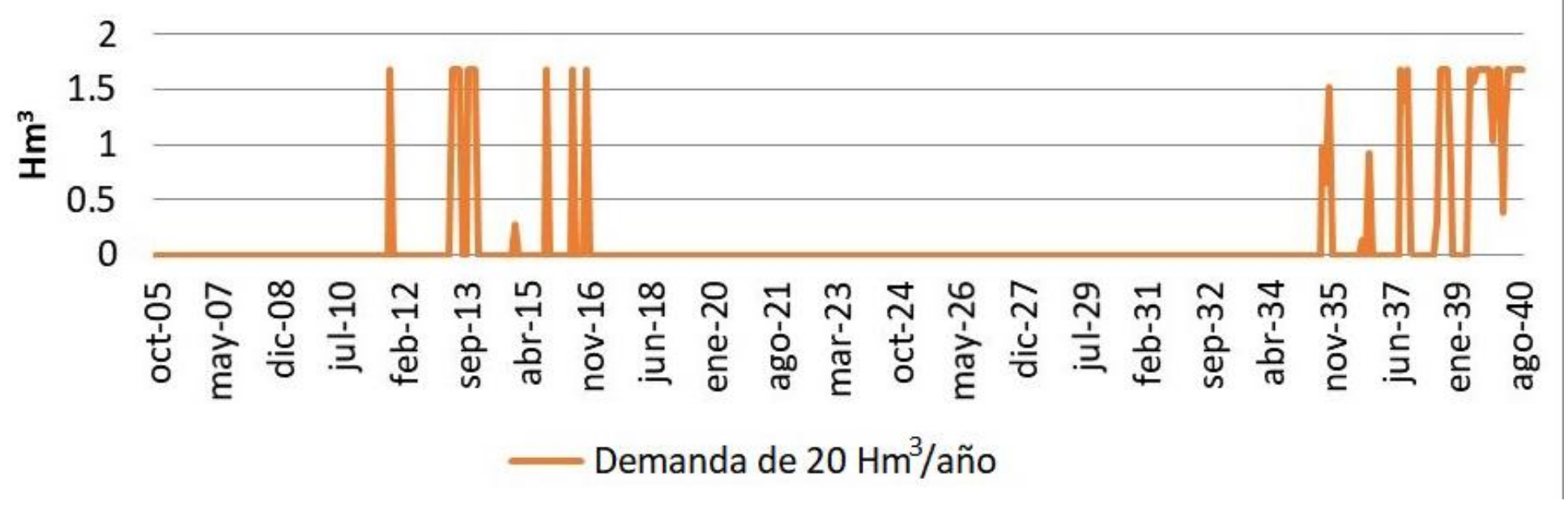

Figura 6. Fallos en la garantía de la nueva demanda de agua para

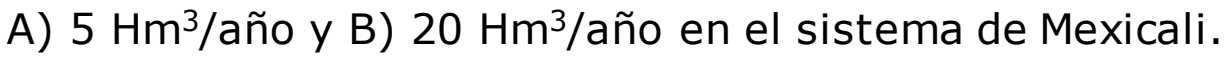

Los fallos en la nueva demanda industrial son relativamente menores para la fase de $5 \mathrm{Hm}^{3}$, pero más significativos en la fase de $20 \mathrm{Hm}^{3}$, manteniéndose similares durante todo el periodo de simulación. Los fallos 
Tecnología y

Ciencias $\stackrel{\Xi}{\Im}$ Aua
2022, Instituto Mexicano de Tecnología del Agua

Open Access bajo la licencia CC BY-NC-SA 4.0

(https://creativecommons.org/licenses/by-nc-sa/4.0/)

presentados a partir del año 2035 coinciden con los fallos en las demandas reales, generándose por las supuestas menores entregas de agua a México. Sin embargo, los fallos entre el periodo 2011 y 2016, en donde el comportamiento del sistema se puede considerar como más histórica por tratarse de un rango de tiempo con entregas de agua observadas, muestran que, en ciertas ocasiones, el sistema de Mexicali no podría cumplir con la nueva demanda industrial al $100 \%$.

\section{Discusión}

Con todos los pasos anteriores es posible llegar a obtener el modelo base para el sistema de flujos de agua en Mexicali, que servirá para evaluar el comportamiento de los flujos en el sistema para cualquier alternativa planteada sobre cambios en la gestión en un periodo de tiempo definido. Sobre este modelo base se puede realizar cualquier tipo de cambios, ya sea en las aportaciones, en las demandas, o en cualquier otro de los elementos del sistema, y obtener la información estadística para poder comparar resultados entre las alternativas. La cantidad, complejidad y tipo de alternativas propuestas a evaluar en el modelo base dependerán de los intereses del usuario. 
Tecnología y

Ciencias $\approx$ Agua
2022, Instituto Mexicano de Tecnología del Agua

Open Access bajo la licencia CC BY-NC-SA 4.0

(https://creativecommons.org/licenses/by-nc-sa/4.0/)

Es importante mencionar que siempre se debe buscar generar un modelo robusto para la gestión de los recursos hídricos. Mientras más datos, periodos más largos e información validada, el modelo base podrá representar mejor la realidad.

La calibración de AQUATOOL normalmente se verifica entre el resultado de algún elemento simulado y los datos observados del mismo elemento, como pueden ser los niveles de una presa, o los flujos en un punto de control en un río o canal. En el caso de este trabajo, no es posible utilizar la presa Morelos en el LIN debido a que el sistema de Mexicali no tiene control sobre ella y, además, agua arriba de esta presa no hay escurrimiento natural, pues todo el río Colorado está controlado por EUA. Ante esta situación y con la ausencia de datos oficiales y públicos de flujos en puntos de control dentro del sistema de Mexicali se puede considerar que el modelo de AQUATOOL funciona correctamente al observarse que las demandas de Mexicali son satisfechas, como sucede en la realidad. Esta situación refuerza la necesidad de poder contar con datos duros oficiales y de buena calidad, para poder ofrecer un análisis confiable en este tipo de investigaciones.

Después de la experiencia de búsqueda, recolección y procesamiento de datos, simulaciones e interpretación de los resultados para el caso de la subcuenca del río Colorado en Mexicali, se proponen los siguientes trabajos futuros que involucran temas desde la disponibilidad de datos hasta nuevos enfoques de análisis para la situación actual y futura. Primero, desarrollar una base de datos con la mayor cantidad de datos posibles y con el mayor periodo disponible para los parámetros meteorológicos, flujos de distribución de agua dentro del sistema, 
Tecnología y

Ciencias $\approx$ Agua
2022, Instituto Mexicano de Tecnología del Agua

Open Access bajo la licencia CC BY-NC-SA 4.0

(https://creativecommons.org/licenses/by-nc-sa/4.0/)

demandas de agua, parámetros físicos del subsuelo e hidrogeológicos, flujos y niveles en cuerpos de agua, extracción de agua subterránea, entregas de agua en el LIN y LIS, datos agrícolas de extensiones, cultivos y aplicación de riegos, eficiencia del riego, tratamientos de agua, retornos al río, etcétera. Segundo, se podría trabajar en una plataforma SIG, en la que se identifiquen todos los elementos, como en el esquema de AQUATOOL, y en los que la información de la base de datos esté contenida, visible y disponible. Tercero, generar y calibrar un modelo base como el presentado en este trabajo, el cual servirá para evaluar el comportamiento desde propuestas para mejorar la eficiencia en el uso de los recursos hídricos hasta para valorar el impacto de cambio climático, o el costo-beneficio de, por ejemplo, proyectos como los propuestos en el Acta 323 para generación de fuentes de agua o algún tipo de reservorios de agua para Mexicali.

Otra demanda que no se está considerando es la de incorporar las necesidades de flujos ambientales al cauce natural del río Colorado estimados por Hinojosa-Herta y Carrillo-Guerrero (2010). Se trata de una cifra entre 390 y $600 \mathrm{Hm}^{3}$ anuales, que requerirían de ajustes en las demandas del sistema de Mexicali. Esta demanda de flujos ambientales causaría mayor competencia por el agua y complicaría el cumplimiento de las garantías de las otras demandas. Si se siguen los ejemplos de otras cuencas en el mundo, la demanda ecológica está en segundo lugar de jerarquía, sólo después de la urbana, lo que resulta en otra razón más para mejorar la eficiencia en los volúmenes consumidos de la demanda agrícola. 
Tecnología y

Ciencias $₫$ Agua
2022, Instituto Mexicano de Tecnología del Agua

Open Access bajo la licencia CC BY-NC-SA 4.0

(https://creativecommons.org/licenses/by-nc-sa/4.0/)

Con los efectos esperados del cambio climático, al aumentar las temperaturas se espera una disminución de los recursos hídricos en la cuenca del río Colorado por evaporación, lo que al mismo tiempo incrementaría las cantidades para satisfacer las demandas debido a las pérdidas en el sistema. Esta condición hace aún más evidente la necesidad de mejorar los sistemas de gestión de recursos hídricos tanto en el ámbito internacional como local en Mexicali.

\section{Conclusiones}

La atención al tema del agua en Mexicali y Baja California (como debería ser en todas las ciudades) es urgente e importante. Aunque en Mexicali aún no se han presentado recortes en los suministros de agua, sí sucede a la fecha en Ensenada, B.C., donde no se puede garantizar el suministro de agua procedente del río Colorado.

Los fallos en la demanda agrícola son difíciles de comprobar al no haber información detallada de las entregas y de los usuarios agrícolas. Lo que sí es una realidad es la actual inconformidad de los agricultores por una variedad de temas al respecto, que incluyen la gestión en el abastecimiento de agua. 
Tecnología y

Ciencias $\approx$ Agua
2022, Instituto Mexicano de Tecnología del Agua

Open Access bajo la licencia CC BY-NC-SA 4.0

(https://creativecommons.org/licenses/by-nc-sa/4.0/)

Agregar una nueva demanda de agua entre 5 y $20 \mathrm{Hm}^{3}$ anuales al sistema de Mexicali podría generar fallos en el sistema de agua superficial, como sucede si se agrega la demanda en el periodo de control. Si bien la nueva demanda industrial presenta fallos en cantidades menores al $10 \%$ de lo que demandaría, es prueba de que su abastecimiento puede estar limitado en ciertos momentos. Además, una demanda de $20 \mathrm{Hm}^{3}$ representa una cantidad significativamente más alta que otras demandas existentes. Incluso una nueva demanda inicial de $5 \mathrm{Hm}^{3}$ debería ser cuidadosamente analizada. Aunque en la actualidad se ha suspendido la instalación de la empresa que requeriría esta nueva demanda, es importante considerar que las necesidades actuales de agua en Mexicali y Baja California pueden no estar garantizadas en un futuro próximo.

No existe una fuente completa de información de datos necesarios para este tipo de estudios. Si bien hay que acudir a las oficinas correspondientes para obtener algunos datos, muchos no los hay o no existen, o no están disponibles para cualquier persona. La cuenca del río Colorado en la parte mexicana presenta poca disponibilidad de datos oficiales y de estudios científicos realizados en la región. Debido a esto se deben hacer importantes asunciones y estimaciones indirectas para poder realizar este tipo de investigaciones. Ello resulta en una disminución en la certeza de los resultados, reduciéndolos a ser estrictamente orientativos.

Lo que es una realidad es que mejorar la eficiencia del sistema de riego del DR-014 y reducir la demanda agrícola; es el área de mayor oportunidad para aumentar la disponibilidad en el sistema. 
Tecnología y

Ciencias $₫$ Agua
2022, Instituto Mexicano de Tecnología del Agua

Open Access bajo la licencia CC BY-NC-SA 4.0

(https://creativecommons.org/licenses/by-nc-sa/4.0/)

A pesar de las posibles reducciones aplicadas a las entregas de agua a México, determinados por el Acta 323, si se mantienen las demandas actuales, éstas podrían ser abastecidas a corto plazo.

La aplicación de AQUATOOL en el sistema de flujos de agua en Mexicali y los resultados obtenidos muestran la utilidad para desarrollar una infinidad de análisis de "¿qué pasaría si...?", como en el caso de agregar la nueva demanda industrial o suponer una modificación las cantidades, etcétera.

La actual situación de conflicto por el agua en Mexicali desde el año 2016 muestran la carencia de comunicación y cooperación entre los usuarios.

\section{Agradecimientos}

Este trabajo se realiza bajo el financiamiento del Consejo Nacional de Ciencia y Tecnología (Conacyt) de México para beca para doctorado en el extranjero otorgada al primer autor, el cual también forma parte de la tesis doctoral.

\section{Referencias}

Adler, R. (2007). Restoring Colorado River ecosystems: A troubled sense of inmensity. Washington, DC, USA: Island Press.

Andreu, J., Solera, A., Capilla, J., \& Ferrer, J. (2007). Modelo SIMGES de simulación de la gestión de recursos hídricos, incluyendo utilización 
Tecnología y

Ciencias $\stackrel{\Xi}{\unlhd}$ gua
2022, Instituto Mexicano de Tecnología del Agua

Open Access bajo la licencia CCBY-NC-SA 4.0

(https://creativecommons.org/licenses/by-nc-sa/4.0/)

conjunta. Versión 3.00. Manual del Usuario. Valencia, España: Universidad Politécnica de Valencia.

Carrillo-Guerrero, Y., Glenn, E., \& Hinojosa-Huerta, O. (2013). Water budget for agricultural and aquatic ecosystems in the delta of the Colorado River, Mexico: Implications for obtaining water for the environment. Ecological Engineering, (59), 41-51.

CEABC, Comisión Estatal del Agua de Baja California. (2017). Informe mensual diciembre 2017. Mexicali, México: Comisión Estatal del Agua de Baja California.

CEABC, Comisión Estatal del Agua de Baja California. (2016). Programa hídrico del estado de Baja California, visión 2035 - Resumen ejecutivo. Mexicali, México: Comisión Estatal del Agua de Baja California.

Christensen, N., Wood, A., Voisin, N., Lerrenmaier, D., \& Palmer, R. (2004). The effects of climate change on the hydrology and water resources of the Colorado river basin. Climatic Change, (62), 337363.

CILA, Comisión Internacional de Límites y Aguas entre México y los Estados Unidos de América. (2018). Entregas de agua a México del Río Colorado. Recuperado de https://cila.sre.gob.mx/cilanorte/index.php/avisos/116entregasagua-rc

CILA, Comisión Internacional de Límites y Aguas entre México y los Estados Unidos de América. (2017a). Acta 323. Aplicación de las medidas de cooperación y adopción de un plan binacional de 
Teçnología y

Ciencias $\stackrel{\Xi}{\triangleleft}$ gua
2022, Instituto Mexicano de Tecnología del Agua

Open Access bajo la licencia CC BY-NC-SA 4.0

(https://creativecommons.org/licenses/by-nc-sa/4.0/)

contingencia ante la escasez de agua en la cuenca del río Colorado. Ciudad Juárez, México: Comisión Internacional de Límites y Aguas entre México y los Estados Unidos de América.

CILA, Comisión Internacional de Límites y Aguas entre México y los Estados Unidos de América. (2017b). Las aguas subterráneas y la jurisdicción de la CILA. Recuperado de http://www.cila.gob.mx/as/baasmxeu.pdf

Cohen, M., \& Henges-Jeck, C. (2001). Missing water, the uses and flows of water in the Colorado River Delta Region. Oakland, USA: Pacific Institute for Studies in Development Environment and Security.

Conagua, Comisión Nacional del Agua. (2018). Estadísticas del Agua en México, edición 2018. Ciudad de México, México: Comisión Nacional del Agua.

Ficklin, D., Stewart, I., \& Maurer, E. (2013). Climate change impacts on streamflow and subbasin-scale hydrology in the upper Colorado River Basin. PLoS ONE, 8(8), e71297.

Gautam, J., \& Mascaro, G. (2018). Evaluation of coupled model intercomparison project phase 5 historical simulations in the Colorado River basin. International Journal of Climatology, (38), 3861-3877.

Hinojosa-Herta, O., \& Carrillo-Guerrero, Y. (2010). Las cuencas hidrográficas de México. La cuenca binacional del río Colorado. México, DF, México: Instituto Nacional de Ecología y Cambio Climático. 
Tecnología y

Ciencias Agua
2022, Instituto Mexicano de Tecnología del Agua

Open Access bajo la licencia CC BY-NC-SA 4.0

(https://creativecommons.org/licenses/by-nc-sa/4.0/)

Howes, D., Burt, C., \& Feist, K. (2012). Basin-wide remote sensing of actual evapotranspiration and its influence on regional water resources planning. Irrigation Training and Research Center, 002. Recuperado de www.itrc.org/papers/pdf/remotesensing.pdf

IPH, Instrucción de Planificación Hidrológica. (2008). Orden ARM/2656/2008, de 10 de septiembre, por la que se aprueba la Instrucción de Planificación Hidrológica (IPH). Madrid, España: Boletín Oficial de España (BOE).

Luecke, D., Pitt, J., Congdon, C., Glenn, E., Valdés-Casillas, C., \& Briggs, M. (1999). A delta once more. Boulder, USA: Environmental Defense Fund.

McKinney, D., Cai, X., Rosegrant, M., Ringler, C., \& Scott, C. (1999). Modeling water resources management at the basin level: review and future directions. Colombo, Sri Lanka: International Water Management Institute.

Medellín-Azuara, J., Mendoza-Espinosa, L., Lund, J., Harou, J., \& Howitt, R. (2009). Virtues of simple hydro-economic optimization: Baja California, Mexico. Journal of Environmental Management, (90), 3470-3478.

Solera-Solera, A., Paredes-Arquiola, J., \& Andreu-Álvarez, J. (2015). AQUATOOL+, Manual de usuario. Valencia, España: Universidad Politécnica de Valencia.

SPABC, Secretaría de Protección al Ambiente de Baja California. (2012). Programa Estatal de Acción ante el Cambio Climático de Baja 
California. Mexicali, México: Secretaría de Protección al Ambiente de Baja California.

Udall, B., \& Overpeck, J. (2017). The twenty-first century Colorado River hot drought and implications for the future. Water Resources Research, 3(53), 2404-2418.

USBR, U.S. Department of the Interior - Bureau of Reclamation. (2019a). Annual operating plan recipients. Recuperado de https://www.usbr.gov/lc/region/g4000/24mo/index.html

USBR, U.S. Department of the Interior - Bureau of Reclamation. (2019b). Sequía en la cuenca del Río Colorado, perspectivas utilizando datos abiertos. Recuperado de https://cidatest.er.usgs.gov/bluedragon/dev/es/

USBR, U.S. Department of the Interior - Bureau of Reclamation. (2016). West-wide climate risk assessments: Hydroclimate projections. Denver, USA: U.S. Department of the Interior - Bureau of Reclamation. 